\title{
The Next Generation European Spallation Source
}

\author{
J.L. Finney \\ ESS Science Co-ordinator, University College, London \\ H. Lengeler \\ ESS Project Leader, CERN, Geneva
}

In 1993 a consortium of research institutions from seven European countries was set up for a technical study of a pulsed spallation source of the next generation, called the European Spallation Source (ESS). In parallel, the scientific needs of neutron scattering methods and instrumentation were also assessed. The studies were supported by the European Community and the European Science Foundation and the final reports covering the results of these studies were released in Spring of this year. ESS has been costed to an accuracy of within $20 \%$ and the technical study has identified further research and development work to complete the database for an engineering design and to reduce the technical risks associated with pushing some parts of the design beyond the present-day technology. Five leading European institutions have agreed to go forward with a 3 years research and development phase.

\section{Condensed Matter Physics}

Neutron scattering provides basic microscopic information on the structure and dynamics of materials, that underpins our understanding of condensed matter, not only in physics but also in fields as diverse as materials science, chemistry, biology, and the earth sciences. It has made outstanding contributions to our detailed understanding on a microscopic level of technically important materials, such as plastics, proteins, polymers, fibres, liquid crystals, ceramics, hard magnets and superconductors, as well as to our understanding of fundamental phenomena such as phase transitions, quantum fluids, and spontaneous ordering.

The neutron is in many ways the ideal probe for the study of condensed matter. It has significant advantages over other forms of radiation in the study of microscopic structure and dynamics which make it complementary to any other powerful techniques. Processes occurring over a wide energy range can be probed, from the neV energies associated with polymer reptation, through molecular vibrations and lattice modes to $\mathrm{eV}$ transitions within the electronic structures of materials.
Structural information can be obtained over distance scales ranging from that of the wavefunction of the hydrogen atom to those of macromolecules.

Neutrons see nuclei, rather than the diffuse electron cloud seen by $\mathrm{x}$-rays, a property which is the basis of many of the techniques that neutrons exploit, for example allowing us to look at the structure of one particular part of a complex sample. The neutron's magnetic moment is ideally suited to the study of the microscopic magnetic structures and magnetic fluctuations that underlie magnetic phenomena in materials. The weak perturbation of the experimental system by the neutron greatly facilitates interpretation, and turns the neutron into a genuine microscopic probe of bulk processes under realistic conditions.

European science is fortunate in having available to it the world's most intense reactor and pulsed spallation neutron sources, namely the Institut Laue-Langevin (ILL) in Grenoble and the ISIS Facility at the Rutherford Appleton Laboratory in Oxfordshire, UK. These two sources provide a complementary pair: they both

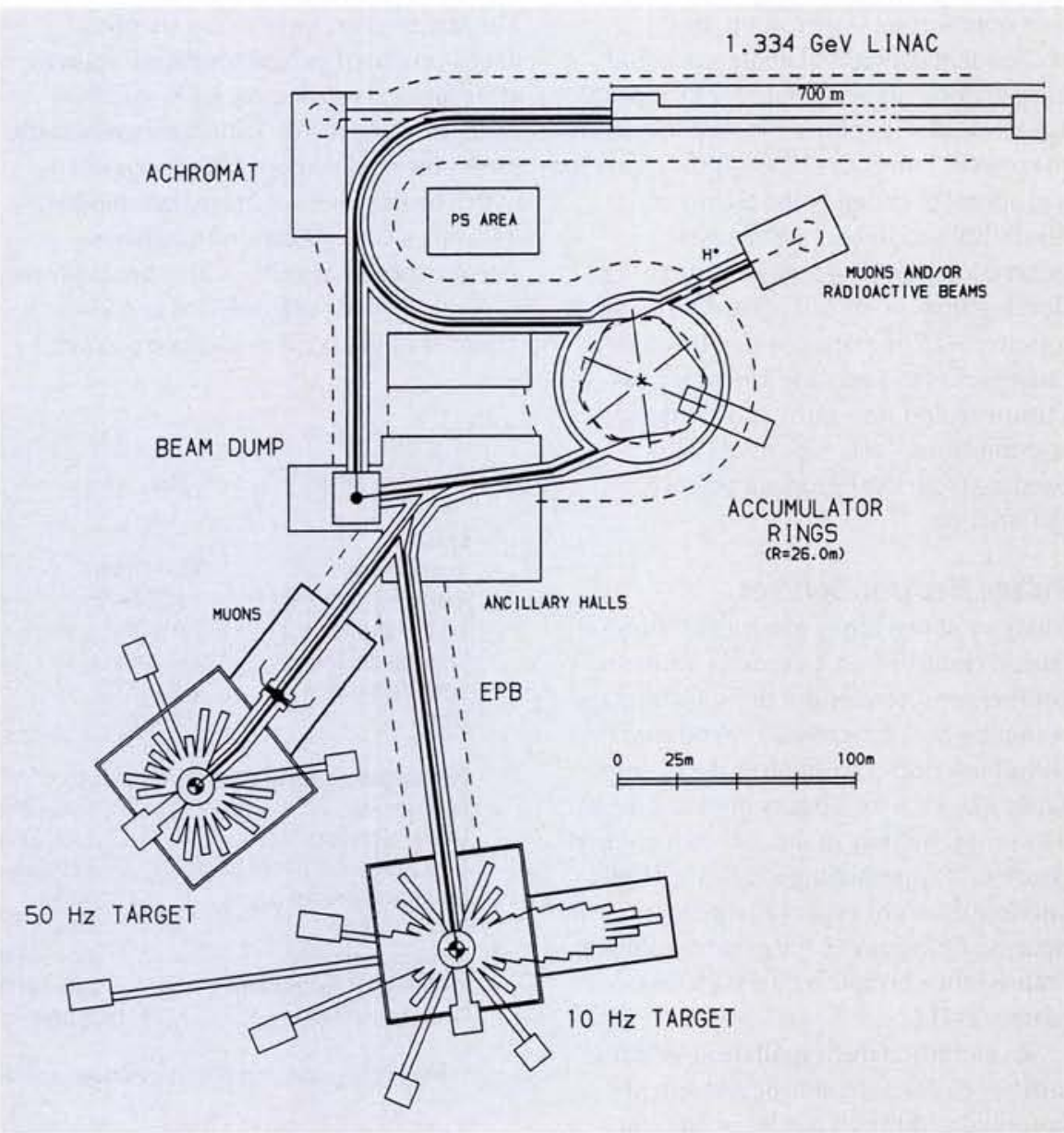


have unique features, but there is also significant overlap in their capabilities. It is not surprising therefore that European neutron scattering research leads the world.

\section{Up Against The Limit}

Despite the major achievements of neutron scattering it is very much an intensity limited technique and present-day neutron experiments are pushing up against the limits of what is possible with today's sources. In many areas, demonstration experiments are being performed that show what may be possible were we able to exploit higher source strengths. Moreover, neutrons are essential if we are to tackle successfully the many problems of increasing complexity that are relevant to the wealth-creating industries. If we are to be able to continue to exploit the unique possibilities of the neutron a more powerful source is essential.

A next generation source is also needed for another very important reason: many of the present-day sources are reaching the end of their technically and economically viable life. This problem was recognised by a panel set up by the European Commission in 1990 which reported that, unless action was taken soon, the consequent drought of neutrons would have serious consequences for European science and its exploitation. Subsequent to the panel's report the CEC approved in 1994 a proposal to examine the technical feasibility and likely cost of a next generation pulsed spallation source. The development of the scientific case for such a source - an operation which involved a large part of the existing and potential European and non-European neutron communities - was supported with funding from the European Science Foundation.

\section{Pulsed Neutron Sources}

There exist two kinds of neutron sources: fission reactors (which are CW neutron sources) and accelerator driven spallation sources where neutrons are produced by the interaction of protons in the energy range of $\mathrm{GeV}$ with a heavy material target. The power density in the core of high flux reactors is approaching a technical limit and one does not expect a large increase of neutron fluxes above the present values as realised for example by the high flux reactor at ILL.

Accelerator driven spallation sources still have a considerable development potential and they have the additional advantage that they can be pulsed. By imposing a time structure on the proton beam, the neutron pulses can be compressed in a short time interval. This does not only increase the peak intensities but one can use time of flight measurements for the determination of incident neutron energies. It avoids the monochromatisation used in CW sources which considerably decreases the neutron intensity at the detector. In this way a large wavelength range can be exploited simultaneously and a more efficient use of neutrons is possible. Time-of-flight measurements ask for very short pulse lengths (ms) and for small repetition rates $(\leq 50 \mathrm{~Hz})$ in order to avoid the overlap of slow neutrons from one pulse with fast neutrons from the next pulse. For beam powers in the range of many $100 \mathrm{~kW}$ or MW this goal cannot be realised today by a linear proton accelerator alone. One therefore considers the use of a pulsed linac combined with an accumulator or an accelerator ring filled by multiturn injection and emptied by fast one-turn extraction for reaching the desired peak power and pulse lengths.

\section{The Most Powerful Source}

The last 20 years have seen a steady development of pulsed spallation sources at Argonne, Los Alamos, KEK, and the Rutherford Appleton Laboratory where the most powerful source (ISIS) is operated with a beam power of more than $160 \mathrm{~kW}$. Recently a CW spallation source has started operation at PSI with a beam power of $600 \mathrm{~kW}$ (to be upgraded to $900 \mathrm{~kW}$ ). There exist plans for even more powerful pulsed sources, in particular in Austria (Austron, 200 to $400 \mathrm{~kW}$ ), KEK, Los Alamos and at the Oak Ridge National Laboratory where a source of $1 \mathrm{MW}$ with a possible later upgrade to $5 \mathrm{MW}$ is under study. The most ambitious project so far is the European Spallation Source (ESS).

\section{The following basic parameters for ESS} have been proposed:

$.5 \mathrm{MW}$ average beam power. This is 30 times the beam power of ISIS and will produce approximately $50 \%$ of the average thermal neutron flux at ILL with peak fluxes more than two orders of magnitudes higher.

$-1 \mathrm{~ms}$ proton pulse length at the targets - Two target stations, one operating at a repetition rate of $50 \mathrm{~Hz}$ up to $5 \mathrm{MW}$ and a second one at $10 \mathrm{~Hz}, 1 \mathrm{MW}$.

\section{Accelerators}

For the reference design a proton energy range between 0.8 and $3 \mathrm{GeV}$ was considered as a compromise between accelerator and target requirements as well as cost. There are a number of ways of meeting the short pulse specifications with a linac and either accumulator rings or rapid cycling synchrotrons. The chosen accelerator option is a $1.334 \mathrm{GeV}$ linac, which develops the full beam power of $5 \mathrm{MW}$ at $50 \mathrm{~Hz}$, followed by two accumulator rings which operate in parallel (fig 1 ).

This combination has been selected because it is considered to be the most reliable option. The linac can be designed to be far away from the space charge limit. The ring layout is simple with constant field
Total beam power

\section{Linac}

Beam energy Average/peak current Repetition rate Beam pulse duration Beam duty cycle Length

\section{$5 \mathrm{MW}$}

$1.334 \mathrm{GeV}(\mathrm{H}-)$
$3.8 / 107 \mathrm{~mA}$
$50 \mathrm{~Hz}$
$2 \times 0.6 \mathrm{~ms}$
$6.0 \%$
$\sim 710 \mathrm{~m}$

\section{Two accumulator rings}

Frequency of parallel operation $\quad 50 \mathrm{~Hz}$ Number of circulating protons per ring $2.34 \times 10^{14}$ Revolution frequency Bunchlength per ring at ejection $\quad 0.4 \mathrm{msec}$ Dual harmonic rf-system $h=1, h=2$ Mean radius of rings $26.0 \mathrm{~m}$

\begin{tabular}{|c|c|c|}
\hline get stations (Flowi & target, horizor & $n, 4$ \\
\hline Beam power & 4 or $5 \mathrm{MW}$ & $1 \mathrm{MW}$ \\
\hline Energy of protons & $1.334 \mathrm{GeV}$ & $1.334 \mathrm{GeV}$ \\
\hline Time structure of proton pulse & $2 \times 0.4 \mathrm{~ms}$ & $2 \times 0.4 \mathrm{~ms}$ \\
\hline Energy content of p-pulses & $100 \mathrm{~kJ}$ & $100 \mathrm{~kJ}$ \\
\hline Repetition rate & $50 \mathrm{~Hz}$ & $10 \mathrm{~Hz}$ \\
\hline Average thermal flux & $7.0 \times 1014 \mathrm{n} / \mathrm{cm} 2 \mathrm{~s}$ & - \\
\hline Peak thermal neutron flux & $2.0 \times 1017 \mathrm{n} / \mathrm{cm} 2 \mathrm{~s}$ & - \\
\hline Decay time of flux & $150 \mathrm{~ms}$ & - \\
\hline
\end{tabular}


magnets and the transverse space charge fields in the rings are relatively low. This layout is also thought to be near to a cost minimum.

The dominating design principle for the accelerators and beam transfer lines is the minimisation of beam losses, guaranteeing maintenance and repair at short notice and with short down-times. Besides small beam losses $(<1 \mathrm{nA} / \mathrm{m})$ the linac has to be optimised for ring injection and this fixes most of its parameters (table 1). Linac losses are reduced by the use of a double front end and a funnel. This is also advisable for the $\mathrm{H}^{*}$ sources which have not yet achieved the required $140 \mathrm{~mA}$ peak currents at a duty cycle of $6 \%$ and with sufficiently low emittance. The front end is followed by a classical drift tube linac and a $700 \mathrm{MHz}$ side coupled linac accelerating particles up to $1.33 \mathrm{GeV}$ with an average accelerating field of $2.8 \mathrm{MV} / \mathrm{m}$. This choice is based on an optimisation of investment and operation cost. By increasing the tune with energy the linac operates in a nonspace charge dominated regime. Extensive particle tracking has been performed and predicts a small number of halo particles which are the dominant sources of particle losses along the linac.

The option for a superconducting, highenergy section for the linac has also been studied although not yet in great detail. A frequency of $700 \mathrm{Mhz}$ and an average accelerating gradient of $8.3 \mathrm{MV} / \mathrm{m}$ is proposed. The reduced length and higher efficiency of the sc version could lead to substantial reduction of investments and operating cost.

Injection into the two rings will be done by $\mathrm{H}^{-} \mathrm{H}^{+}$charge exchange using a thin foil. The injection scheme uses simultaneous 'painting' in the longitudinal and both transverse phase planes and reduces the average number of foil transversals (an important source of injection losses) to below 10. For sufficiently low injection losses the halo of the injected $\mathrm{H}^{-}$beam has to be carefully controlled and removed. A $180^{\circ}$ achromatic bending region for momentum and transverse beam collimation is introduced in the transfer line between linac and rings. Extensive simulations have shown the viability of this design.

For the two accumulator rings, which have to handle each $2.35 \times 1014$ circulating protons per bunch, the minimisation of losses around the rings and an efficient collection of losses in the injection and extraction region is crucial. Design values for losses are set to $500 \mathrm{~W}$ per ring. The ring lattice contains six bends and six straight sections housing the rf, injection, extraction and beam collimation systems. A switch yard allows channelling the extracted beam to the two target stations. At the target an elliptical beam cross section $(200 \times 60 \mathrm{~mm} 2)$ is proposed.

The possibility for using the long linac pulse $(1.2 \mathrm{~ms})$ in combination with a third dedicated target station has been considered but not worked out in detail.

\section{Targets}

Targets have to withstand not only the large average beam power of $5 \mathrm{MW}$ but also very short proton pulses ( $1 \mathrm{~ms}$ ) with an energy content of $100 \mathrm{~kJ}$. Consequently the deposited energy is relaxed predominantly elastically and produces shock waves in the target material. Targets are therefore submitted to a combined load of high radiation damage, large temperature gradients and stress waves. After an initial investigation of a water cooled Ta-target, studies were concentrated in a liquid metal target with horizontal beam injection. There are numerous arguments in favour of a liquid target. Liquids are not affected by radiation damage and thermal cycling and stress waves are confined to the target window and the target container. The absence of water cooling allows higher neutron yields and avoids electrolysis, corrosion and tritium production linked to the water circuits. Altogether a longer lifetime and a greater potential for high beam power is expected.

Mercury was chosen as a radically new target material instead of the earlier candidates $\mathrm{Pb}$ and $\mathrm{Pb}$ - $\mathrm{Bi}$. Its rather high thermal neutron absorption cross section is of no major concern for a pulsed source with short neutron pulses. An assessment of the hydrodynamical, thermal and neutronic properties of $\mathrm{Hg}$ and of material problems for windows and containers has indicated the basic feasibility for a pulsed, 5 MW source. Neutron yield calculations have shown that an increase of $30 \%$ can be expected as compared to a water cooled Taplate target with significantly less problems from afterheat.

For a beam power up to $5 \mathrm{MW}$ water and supercritical hydrogen are the candidate moderator materials. Four basic types of moderator have been considered:

- A high resolution ambient temperature moderator

- A high intensity ambient temperature moderator

- A high intensity cold moderator

- A high resolution cold moderator
A wide range of material and geometry combinations for the target-moderatorreflector block has been explored. $\mathrm{A} \mathrm{Pb}$ reflected target system with 4 moderators is preferred to a beryllium reflected system; it gives, for example, a peak thermal neutron flux of $2 \times 1017 \mathrm{~cm}-2 \mathrm{~s}-1$ with a decay time of $\mathrm{t}=150 \mathrm{~ms}$ and an average flux of $7 \times 1014 \mathrm{~cm}-2 \mathrm{~s}-1$.

The design of the ESS target stations ( fig 2) has evolved gradually to a modular and flexible layout so that the future requirements of users can be reasonably accommodated. In addition, the layout should allow either a Hg or a Ta-plate target to be used. The $5 \mathrm{MW}$ and $1 \mathrm{MW}$ target stations are to be made as similar as possible. A layout with horizontal removal of the target, including its primary cooling circuits and a vertical removal of the moderator-reflector units, has been proposed. This layout limits the position of neutron beam tubes to an angular range of about $2 \times 120^{\circ}$. A total of 18 beam holes, some equipped with multiple neutron guides, are foreseen at each target station.

The design allows beam lines to be changed so that they can accept neutrons from a bottom or top moderator without major changes of the target block. All operations involving the handling of potentially activated or contaminated equipment are performed in a controlled enclosure, physically separated from the user areas. For the target as well as for the accelerator layout great attention has been given to high availability and reliability. This is crucial for an installation where more than 2000 experiments per year will be performed.

\section{The Scientific Potential}

The ESS will deliver a raw neutron intensity of up to 30 times that of ISIS, presently the world's most powerful such source. With appropriate development in neutron instrumentation, advances in data rates of up to three orders of magnitude are anticipated in some fields. This effective neutron intensity gain will be used in a variety of ways, with trade-offs possible between intensity, resolution, sample size and 'exposure' time. For example, trading increased intensity for higher resolution will enable accurate and precise structure refinements on the increasingly complex systems that are central to modern materials science. The ability to measure weaker signals will allow us to study details of the subtle effects and small changes that are seen to be increasingly important in controlling the behaviour of new materials 
(eg high temperature superconductors). Shorter exposures will allow us to measure structure and dynamics over a much wider parameter space than is presently possible. Following variations with pressure, temperature, magnetic or electric field, and concentration etc will allow us to solve real problems, rather than be restricted to single experiments at a single state point that shows us only a small fraction of the picture. Shorter time measurements will also allow real-time structural and dynamic studies of chemical reactions, including in situ catalysis. Kinetic processes and materials behaviour under non-equilibrium conditions can be followed. A dramatic reduction in the unit cost of certain neutron measurements will open up the technique to routine industrial exploitation. High quality measurements will become possible on smaller systems either inherently small such as a crack tip or a thin layer, or available only in small quantities such as new materials, isotopically enriched samples, or dilute solutions. Experiments under extreme sample environment conditions also offer the prospect of new and exciting science.

\section{Questions Answered}

In areas other than condensed matter, ESS will allow us to perform a number of unique experiments which will help to determine the basic structure of the elementary interactions in nature, to elucidate the history of the Universe, and to study a number of questions in quantum and measurement theory. An answer to the question of the handedness of nature which could be obtained through a clearcut 'yes' or 'no' experiment on ESS - could open up exciting new physics beyond today's standard model, while an improved understanding of the strong force will address questions of matter-antimatter symmetry and of Grand Unification.

The ESS can also produce high intensities of a variety of other particles. Through the implementation of an ultraslow muon beam, surfaces and thin layers will be opened up to the sensitivity and selectivity of muon spectroscopy.

Neutrinos could be made available at an unprecedented intensity to open up a new era in neutrino physics, with strong impact on particle physics, astrophysics, and cosmology. A radioactive nuclear beam facility could be exploited in both fundamental and applied studies. The former include nuclear structure studies as the neutron/proton ratio is varied, and the properties of unstable nuclei that are important in nuclear astrophysics. Possible applications include ion implantation studies relevant to semiconductor microelectronics and advanced metallurgy, tumour radiotherapy, and the provision of the nuclear data needed for effective nuclear waste transmutation.

ESS: A New Generation Neutron Source for Europe. Vol. II: The Scientific Case, ISBN ogo 2376608. Vol. III: The ESS Technical Study, ISBN 0902376 659. Contact: J. Kjems, Chairman ESS Council, Risø National Laboratory, P.O. Box 49, DK - 4000 Roskilde

Fig 2 The layout of the target station

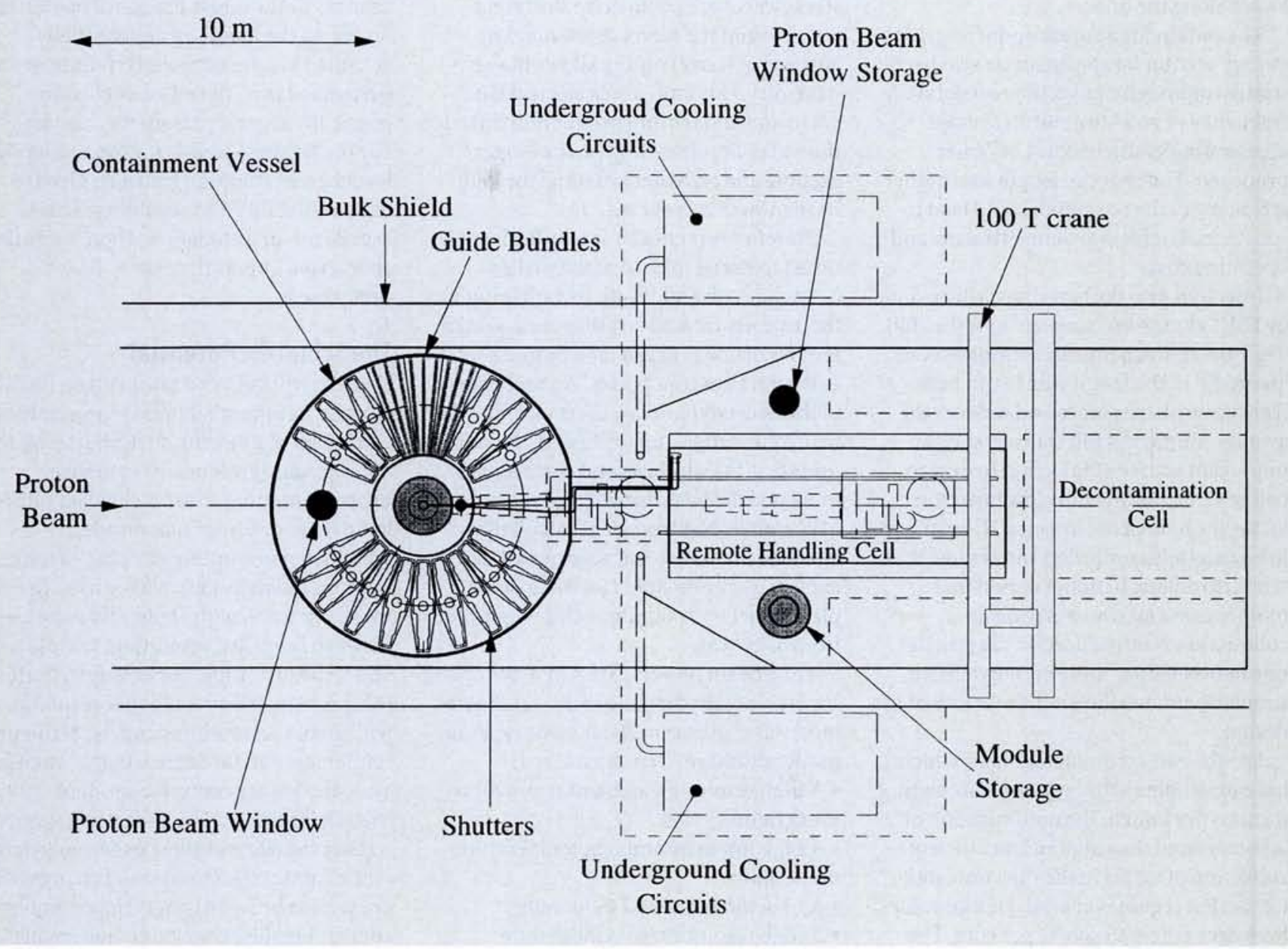

